\title{
La révolution au village, avec Saint-Just, d'après le registre des délibérations communales de Blérancourt
}

\section{Bernard Vinot}

\section{(2) OpenEdition Journals \\ Édition électronique \\ URL : https://journals.openedition.org/ahrf/1341 \\ DOI : 10.4000/ahrf.1341 \\ ISSN : 1952-403X \\ Éditeur : \\ Armand Colin, Société des études robespierristes}

Édition imprimée

Date de publication : 1 mars 2004

Pagination : 97-110

ISSN : 0003-4436

\section{Référence électronique}

Bernard Vinot, «La révolution au village, avec Saint-Just, d'après le registre des délibérations communales de Blérancourt », Annales historiques de la Révolution française [En ligne], 335 I janviermars 2004, mis en ligne le 20 février 2008, consulté le 23 avril 2022. URL : http:// journals.openedition.org/ahrf/1341; DOI : https://doi.org/10.4000/ahrf.1341

Ce document a été généré automatiquement le 23 avril 2022.

Tous droits réservés 


\title{
La révolution au village, avec Saint- Just, d'après le registre des délibérations communales de Blérancourt
}

\author{
Bernard Vinot
}

1 Le cadre n'a guère changé en deux siècles. Les rues n'ont plus le même nom, mais le noyau historique du bourg est aujourd'hui conforme à ce qu'il était au XVIII ${ }^{e}$ siècle. L'essentiel de la dramaturgie révolutionnaire s'est déroulé sur un triangle d'un kilomètre de côté dont les sommets seraient le château, l'église, la maison de Saint-Just et les lieux forts, la place du Marais (actuellement du Général Leclerc), où se déroulent les manifestations de la garde nationale, l'église, seul bâtiment assez vaste pour accueillir les assemblées plénières de la commune, la halle, centre des marchés et cœur de la vie économique.

2 La révolution au village est consignée dans les délibérations communales qui couvrent une période de cinq ans, très exactement du 7 mars 1788 au 30 mai 1793 et dont l'existence est connue depuis longtemps. Plusieurs amateurs d'histoire les ont consultées et en font état aux $\mathrm{XIX}^{\mathrm{e}}$ et $\mathrm{XX}^{\mathrm{e}}$ siècles, mais ils se sont contentés d'en extraire ce qui leur apparaissait comme les morceaux de bravoure, ainsi que l'on pratiquait à cette époque, voire d'en reproduire certains, comme l'a fait Charles Dessin dans une publication d'intérêt local ${ }^{1}$. Puis ces registres qui méritaient une analyse sérieuse ont longtemps disparu. Dans les années 1970-1980, on en ignorait le sort. Heureusement, ils sont maintenant conservés aux archives départementales de l'Aisne ${ }^{2}$.

3 Ce dépôt se présente sous la forme de trois registres de respectivement 34, 86 et 46 feuillets au format $23 \times 36 \mathrm{~cm}$, soit au total 332 pages dont 49 sont consacrées aux vingttrois premiers mois (mars 1788-janvier 1790) sans Saint-Just (moyenne : 2,1 pages par mois), 276 aux trente et un mois suivants (janvier 1790-septembre 1792) avec Saint-Just (moyenne : 8,9 pages par mois) et quarante-neuf aux neuf derniers mois (septembre 
1792-mai 1793) sans Saint-Just (moyenne : 5,4 pages par mois). Cette simple observation laisse présumer la place occupée par ce jeune homme de vingt-trois ans que sa minorité légale met théoriquement hors jeu politique. Nous allons pourtant le voir directement s'immiscer dans les affaires de la commune avant de nous interroger sur la nature, l'intérêt et la portée de son influence.

I - La conquête du pouvoir municipal par les partisans de Saint-JustLes « notables » de Blérancourt avant la Révolution

4 Un an donc avant la Révolution apparaissent au bas de la transcription des délibérations communales les signatures d'une élite sociale, entendons par là le groupe de ceux qui participent à la gestion publique du village. On y distingue des "lettrés ", des propriétaires terriens et un groupe beaucoup moins homogène de commerçants et d'artisans.

5 Les « lettrés » tiennent une place importante dans la mesure où ils sont généralement rédacteurs de suppliques, de courriers officiels ou d'actes notariés, ce qui leur donne du prestige auprès de leurs compatriotes pour la plupart analphabètes. L'un des plus en vue est Antoine Gellé, cinquante-huit ans en 1788. Issu d'une famille de laboureurs de Guiscard dans le Noyonnais, il est arrivé à Blérancourt à l'âge de quinze ans, y a passé toute sa vie professionnelle, exerçant successivement ou simultanément plusieurs activités : notaire, marchand de bois et surtout régisseur de la seigneurie, procureur fiscal de sa justice. Il vit à proximité du château avec sa troisième épouse dont il a reconnu la fille bâtarde Thérèse. Caractère irascible, cassant, il trafique brutalement de l'influence que lui donne sa position. Myope dans son temps, allergique aux changements, il sera l'adversaire farouche de Saint-Just mais aussi un bretteur encombrant pour les siens, tant il se déconsidère auprès de la population par ses excès. Dans le même camp, François Thorin, 61 ans, fils de laboureurs aisés du gros bourg d'Attichy, proche de Compiègne, est autrement adroit et redoutable. Successivement notaire, lieutenant de justice seigneuriale, vérificateur des actes, il est compétent, modéré, conciliant, expert dans l'art de déjouer les bottes de ses adversaires, c'est un homme influent sous tous les régimes. Ce milieu de robins est endogame : le fils Thorin, François-Emmanuel, successeur de son père à l'étude, a épousé Thérèse Gellé ; la fille Thorin a épousé Emmanuel Decaisne, un notaire issu d'une vieille famille de Noyon animatrice de la franc-maçonnerie locale. On peut assimiler à ce groupe François Binant, fils et frère de fermiers installés sur les terres riches du Soissonnais, vivant bourgeoisement, c'est-à-dire de ses rentes, et dont la signature terminée par un paraphe prétentieusement alambiqué montre qu'il a eu accès à l'instruction, probablement chez les Oratoriens de Soissons; le syndic Claude Nicolas Carbonnier, chirurgien, considéré pour avoir joué un rôle de premier plan lors de la terrible épidémie qui a frappé le bourg en cette année 1788 et s'être dévoué sans limite à la détresse des petites gens ; enfin, quoique de situation beaucoup plus modeste, François Lesassière, 41 ans, né à Blérancourt, fils du concierge du château, chargé d'une nombreuse famille, évoluant dans la mouvance de la seigneurie qui lui assure la garde de ses chasses et le greffe de la commune.

Le deuxième groupe est constitué de quatre des cinq laboureurs alors recensés au village : Louis Honnoré, 64 ans, Pierre Dremont, 35 ans, Jean Borgne, 45 ans, Jean Eloi Wargnier, 54 ans, cultivant respectivement 46, 69, 129 et 139 arpents de terre ${ }^{3}$, tous natifs de Blérancourt, pratiquant aussi l'endogamie dans le milieu des fermiers du 
Soissonnais. On trouve également à leurs côtés Brice Fillion, 40 ans, pourvu seulement d'un peu moins de neuf arpents, mais qu'il cultive intensivement en jardinage.

Enfin, au nombre des commerçants et artisans on relève les signatures d'Augustin Dutailly, 57 ans, marchand de draps mais auparavant greffier en chef de la justice seigneuriale, le meunier Antoine Meresse souvent soupçonné de tricher sur le poids de farine qu'il rend à ceux qui apportent au moulin le grain à moudre, deux autres marchands, Jean Rigeasse, 75 ans, Théodore Massy, 44 ans, François Monneveux ancien cuisinier du château de Cuts, installé aubergiste à Blérancourt depuis 1781 et Joseph Ramart, 58 ans, serrurier, époux d'une Carbonnier dont le gendre Victor Thuillier, 23 ans, est le fidèle ami de Saint-Just. La situation économique des membres de ce groupe va des limites de la pauvreté à l'honnête aisance. Dutailly qualifié ici de commerçant gérera avec ses deux fils à la fin de l'Empire une petite fabrique de textile. Beaucoup sont sensibles aux idées des Lumières. Ils présentent des similitudes, mutatis mutandis, avec les animateurs de la future sans-culotterie parisienne.

Ces notables, tous en leurs spécialités, décident au nom d'une population de neuf cent cinquante habitants répartis en cent quatre-vingts foyers dont soixante-quatre sont propriétaires d'une terre (inférieure à un arpent pour vingt-six d'entre eux). Les autres, soit près des deux tiers, vivent dans la précarité avec pour première préoccupation la nécessité de se nourrir quotidiennement. Les états de recensements les désignent comme tisserands, fileuses, manouvriers, lingères, mandeliers, cordiers, mulquiniers, jardiniers, désignations floues que seule la pratique d'archives recoupées peut éclairer : celle de jardinier par exemple qualifie à la fois un habitant aisé, comme Alexandre Debrie, faisant valoir une propriété de près de quatorze arpents en culture intensive, un propriétaire de moins d'un arpent comme Pierre Patry, mais en même temps employé à temps plein sur les jardins du château, confondant de ce fait ses propres intérêts avec ceux de son maître ou le pauvre hère donnant un coup de main ici et là en saison. Beaucoup ne signent pas leur nom, peu savent écrire, aucun n'est capable de rédiger une supplique. Sans avoir et sans savoir, ces démunis sont dans la dépendance de la paroisse et de la seigneurie. Il n'est pas rare que la cure leur avance les fonds nécessaires aux achats de filasses qui seront façonnées durant tout l'hiver. De son côté le château peut à discrétion pourvoir ou non en avantages ou en petits emplois de police, de justice ou de gestion des marchés. Les procureur, lieutenant, greffier, les surveillants de poids et mesures, les receveurs de taxes, les jardiniers et cultivateurs bénéficiaires de baux profitent jalousement de ces miettes de privilèges non sans susciter du même coup le vent mauvais de l'envie et de la jalousie.

Il n'est pas impossible de déceler dans ce microcosme des coalitions d'intérêts, comme ce 1er juin 1789 où l'on voit trois laboureurs et un jardinier ${ }^{4}$ proposer un changement du rôle d'imposition, probablement dans le sens d'une définition plus favorable aux possédants. Mais on n'en sait pas plus, sinon que leur tentative est rejetée par soixantesept de leurs compatriotes. Il faut du reste constater que tous les propriétaires importants ne se solidarisent pas avec cette minorité de requérants. C'est sans doute que certains ont de bonnes raisons d'être prudents par rapport aux puissants. D'une façon générale, les réactions sont plus passionnées que raisonnées en fonction de rancunes recuites ou de comportements s'insérant au contraire dans le cadre de cercles de convivialité, d'alliances matrimoniales, de voisinages et d'amitiés qu'attestent les signatures conquérantes ou maladroites au bas des actes portés sur les registres paroissiaux ou qui se manifestent sélectivement entre habitués chez chacun des douze 
aubergistes du bourg. Il y a pourtant, plutôt chuchoté que franchement débattu à cette époque, un sujet qui fâche.

10 Avec le grand mouvement physiocratique qui s'est amorcé au milieu du siècle, le château a pratiqué des défrichements et des plantations forestières sur les "communaux», ces terres peu exploitées parce que marécageuses ou escarpées, laissées par tradition à la disposition de tous et particulièrement utiles aux pauvres. $\mathrm{Ce}$ phénomène d'accaparement a pris une particulière ampleur depuis que Grenet a acheté la seigneurie en 1782 avec l'intention de la rentabiliser au maximum. Le problème est soulevé dans la séance du 27 avril 1788, soit plus d'un an avant les premiers événements révolutionnaires à Paris. On apprend qu'un mémoire a été envoyé au châtelain "concernant la restauration des biens communaux de la paroisse ». La question est ainsi portée indirectement sur la place publique par une interpellation du seigneur demandant si ce mémoire a fait l'objet d'une délibération municipale. Le syndic Carbonnier, embarrassé, doit avouer que l'envoi a été fait à la seule initiative de Louis Honnoré, sans que ses collègues soient au courant. Les spoliations à leur profit de certains propriétaires du village qui ont imité le seigneur peuvent expliquer cette absence d'unanimité. Mais en dépit de cette mésentente, l'affaire prend suffisamment de consistance pour que le syndic Carbonnier fasse appel à la «Commission Intermédiaire $d u$ Soissonnais", instance expérimentale de conciliation entre le pouvoir régional (la généralité de Soissons) et les communes. Par chance pour notre information, la procédure permet de recueillir cette description de la municipalité de Blérancourt: "L'assemblée est composée entre autres de sept personnes qui sont suspectes à la communauté : le sieur Gellé, régisseur de la terre, le sieur Thorin, son fils gendre du sieur Gellé et représentant du seigneur, les sieurs Warnier, Borgne et Fillion, fermiers du seigneur et le sieur Lesassière, greffier, son garde-chasse. Que tous ces membres dépendent du sieur Gellé et ne pouvaient qu'adhérer à ses propositions dans la crainte d'éprouver le même sort que le sieur Dremont auquel le sieur Gellé avait ôté une ferme parce qu'il voulait faire rentrer des fonds communaux dont le seigneur s'était emparé » 5. La situation apparaît tellement explosive à la très modérée commission qu'elle se résout à provoquer une décision ministérielle imposant la démission de Gellé en septembre 1788. Dès lors, en marge de l'effervescence parisienne, il ne se passe plus grand chose à Blérancourt jusqu'au lundi 7 décembre 1789.

L'épisode malencontreux de la Saint-Nicolas

11 Ce jour-là un groupe de jeunes gens, dont certains sont armés de fusils et d'épées, extorquent au syndic Carbonnier un prix imposé sur les grains, notamment sept livres l'essain ${ }^{6}$ de froment au lieu de neuf au marché précédent. Cet attentat à la liberté des prix provoque l'indignation des laboureurs et des vendeurs qui dénoncent en outre les voies de fait dont se sont rendus coupables les agresseurs, y compris sur des femmes. On ne saura probablement jamais si Saint-Just est, ou non, à l'origine de cette action. Les accusations de Gellé incitent plutôt à en attribuer la paternité à Villain Daubigny ${ }^{7}$. Mais il faut bien reconnaître qu'en terme d'efficacité révolutionnaire elle est non seulement sans lendemain, mais totalement contre-productive. Elle déclenche l'indignation des marchands venus souvent de loin, la désapprobation de la majorité des acheteurs craignant que leur marché ne soit transféré au bourg voisin de Coucy-leChâteau ${ }^{8}$; elle provoque le grand retour de Gellé et lui donne occasion de fonder une milice dont il écarteçceux qui par leur mauvaise conduite sont incapables d'y être admis " ${ }^{9}$. Il en prend le commandement en chef et nomme capitaines sous ses ordres 
Binant, Decaisne et Thorin jeune, soit un rentier et deux notaires. En s'appuyant sur une garde ainsi recrutée à sa mesure et épurée, le vieil aristocrate reprend la main.

Le grand tournant : les 30 et 31 janvier 1790

12 Samedi 30 janvier à quatorze heures, la municipalité réinvestie par Gellé prend la décision de convoquer les habitants, le lundi suivant 1er février à neuf heures, afin d'élire, conformément aux directives ministérielles, une nouvelle municipalité. La délibération prévoit pour le lendemain, dimanche 31, la pose d'affiches invitant à l'assemblée les citoyens actifs « avec prohibition à toute autre personne de s'y trouver » 10. Mais ce 31 précisément, avec la complicité du syndic Carbonnier, est lancée une fulgurante contre-attaque par les adversaires de Gellé qui font procéder aux élections en s'appuyant sur l'argutie juridique suivante: le décret de l'Assemblée nationale sanctionné par le roi demandait de procéder aux élections sous huit jours après sa publication. Or, cette publication a été faite le dimanche 24 , on peut donc considérer que le huitième et dernier jour est précisément le dimanche $31^{11} \ldots$

13 Avec une audacieuse détermination les conjurés rassemblent quatre-vingts électeurs, élisent un maire, dix-sept officiers municipaux et douze notables, puis incontinent, mettent en place une nouvelle milice bourgeoise encadrée par des sympathisants. Cinquante-trois signataires approuvent ces opérations. On n'est guère étonné de relever au nombre des élus les noms d'Honnoré, maire, Monneveux, procureur, Augustin Dutailly, Carbonnier, ni d'assister à la promotion de Victor Thuillier au greffe, tous contestataires de l'Ancien Régime, mais on observe que, par rapport au mois précédent, Decaisne (veuf en premières noces qui va épouser Louise de Saint-Just dix jours plus tard) nommé colonel de la milice, et Binant nommé capitaine, ont changé de camp. Cette nouvelle équipe est de composition sociale plus modeste que la précédente. Elle ne compte qu'un seul laboureur et aucun des nouveaux élus, notables ou non, n'est inféodé à la seigneurie. On ne trouve trace de Saint-Just ni dans les promus, ni dans les signataires. À croire qu'il est absent. En fait, le jeune homme se sait surveillé et, n'étant pas majeur, il veut avant tout éviter d'offrir à ses détracteurs l'occasion de faire annuler cette entreprise à la hussarde. Mais l'observation attentive de la transcription de la délibération montre que le coup de force est réalisé avec sa participation active : les treize premières lignes et la mention en marge : "acte de formation de la municipalité le 31 janvier 1790 » sont en effet écrites de sa main avant que Victor Thuillier ne prenne à son tour la plume. On trouve par ailleurs d'autres passages autographes, notamment une recommandation sur les modalités de suppléance à la milice bourgeoise signée Honnoré, Carbonnier et autres..., un réquisitoire au sujet d'un déboisement communal ${ }^{12}$, attribué au procureur de la commune et signé Monneveux, ainsi qu'une feuille volante que Thuillier a intégralement recopiée en complément du rapport sur le contentieux des communaux du 17 octobre $1790{ }^{13}$. On se doutait bien que le futur Conventionnel inspirait les paysans de son village, ces documents en apportent la preuve et la méthode.

Enfin, la prise du pouvoir municipal est sanctionnée vingt jours plus tard par une originale manifestation de serment civique. Saint-Just a écrit lui-même au registre: "Ce jourd'hui 21 février 1790 la commune de Blérancourt sans distinction d'âge, de sexe et de rang a prêté le serment civique et juré fidélité à la loi, au roi et à la constitution et tous ont signé ». Suivent quatre-vingt-onze signatures dont, pour la première fois celle de Saint-Just, peu compromettante en l'occurrence, puisqu'elle témoigne d'une réunion dont le caractère unanimiste est affirmé et à laquelle ceux qui 
n'ont pas participé seront regardés comme de mauvais citoyens. Bonne occasion donc de repérer les partisans du jeune homme, mais surtout de relever la signature de quatorze femmes dont la mère et les deux sœurs du futur Conventionnel ${ }^{14}$. Une manifestation de cette nature, à ce moment-là, est probablement unique en France. Elle est très éloignée de ce que sera cinq mois plus tard la Fédération nationale de conception fayettiste. Qu'elle puisse accueillir plus de $15 \%$ de femmes est particulièrement remarquable dans la mentalité du temps empreinte de préjugés antiféministes, même chez les révolutionnaires avancés. Elle témoigne clairement de la position de Saint-Just sur cette question. En contrepoint, le procès-verbal de la fête de la Fédération à Blérancourt réunira le 14 juillet 1790 cent-cinquante signatures ${ }^{15}, \mathrm{y}$ compris celles du curé, des Révérends Pères Feuillants, du fondé de pouvoir du seigneur, mais aucune apposée par une femme.

II - Limites de l'influence de Saint-Just

Les partisans du changement sont très minoritaires au village surtout lorsque les conditions d'âge et de cens inscrites dans les dispositions constitutionnelles sont appliquées avec rigueur. Très vite, ils se heurtent aux autorités religieuses influentes sur le comportement de nombreux fidèles. Or, l'Église tient à Blérancourt une place importante puisque, en plus de la paroisse de Saint-Pierre-aux-Liens, le village compte un monastère de Feuillants et un " hôpital » - c'est-à-dire un établissement d'éducation pour les pauvres orphelins - qui en dépend. Les membres de la communauté affichent pour la plupart une piété de façade jusque dans les débuts de la Révolution: les manifestations publiques se déroulent souvent en présence du curé Flobert et les occasions ne manquent pas de chanter un Te Deum ou le Domine salvum fac regem. À l'occasion de la mise à la disposition de la Nation du couvent, Saint-Just et les siens vont pouvoir mesurer l'hostilité dont est l'objet la politique religieuse de la Constituante et les complicités locales dont disposent les Pères.

16 À la date du 7 mai 1790, une description des revenus et un inventaire de ce monastère occupent les huit premiers feuillets du 2e registre, rédigés par Thuillier avec quelques surcharges, semble-t-il, de la main de Saint-Just. Hélas, les municipaux doivent se rendre à l'évidence et déplorer les multiples disparitions : «[...] nous croyons devoir observer [dictent-ils au greffier] qu'il existait il y a un mois environ un cheval, deux vaches [...] quinze à vingt couverts d'argent [...] quinze à vingt paires de draps, dix douzaines de serviettes [...] douze matelas [...] », mais ils ne peuvent qu'en constater la disparition. Se sont volatilisés aussi les cuivres, les étains, les faïences, alors que "l'ordre faisait l'achat tous les trois ans de deux caisses de faïences dans le nouveau goût tant en plats qu'en assiettes, tasses à café et autres objets». Plus de tables en marbre, ni de dessus de tablettes en marbre ni d'encoignures. Les chaises et fauteuils en merisier de la salle à manger ont été remplacés par un mauvais ameublement. Deux crédences et deux tables de marbre ne sont plus dans la chapelle, " pourtant tous ceux qui ont assisté dimanche dernier à la messe des R.P. Feuillants les ont encore vues ». «Soustraits" encore plusieurs tableaux dans leurs cadres dorés dont certains de valeur. Plus de vin dans la cave, alors que les religieux avaient encore augmenté leur réserve " par l'achat de deux à trois cents bouteilles, tant vin de Beaune rouge que vins blancs, planquette [sic] de Limoux et Chablis à eux adjugés il y a environ dix-huit mois à l'inventaire du sieur Le Borgne décédé chanoine de Noyon ». Même plus de bouteilles vides... ! Cette litanie de regrets laisse percevoir les connivences efficaces dont a pu bénéficier l'établissement religieux. Le rapport de cette inspection se termine sur 
l'espoir que, dans l'intérêt d'une population éprouvée, ce monastère soit réhabilité en une manufacture pourvoyeuse de prospérité. Cet espoir-là sera tout aussi déçu !

La municipalité sait néanmoins faire preuve d'autorité pour exiger la prestation du serment civique de la part des sœurs de l'Enfant-Jésus qui desservent «l'hôpital ». Dès qu'elles sont sollicitées le 16 mai 1791, elles demandent un délai pour en référer à leurs supérieurs. L'une d'elles s'exécute de son plein gré le 22 mai, mais subit les persécutions de ses consœurs âgées. Finalement toutes prêteront le serment le 14 juin, à l'exception de la " première » qui déclare être assez vieille pour être en retraite ${ }^{16}$. Le 17 septembre 1791, la municipalité décrète aussi le chant d'un Te Deum en action de grâce de ce que le roi a signé la Constitution. « Ordre sera donné à $\mathrm{M}$. le curé Flobert de se conformer à la présente délibération ", ce qui n’a pas dû lui être agréable...

Ici comme ailleurs les rapports entre les révolutionnaires et l'Église se sont très rapidement tendus. Il n'est pas fait mention au registre de violences physiques ou verbales. On peut néanmoins penser qu'elles n'ont pas manqué à la lumière d'un témoignage postérieur, mais mettant en cause des survivants de l'entourage de SaintJust. Dans une lettre de dénonciation adressée au duc de Berry en date du 14 juillet 1815 et intitulée: "individus dangereux de la commune de Blérancourt», le royaliste Beaumé, qui avait été momentanément incarcéré à la Conciergerie en l'an II, accuse notamment Eloy Lefèvre d'avoir déclaré "qu'il assassinerait les prêtres même à l'autel » et Isidore Defente «fabricant de coton, habitant le presbytère de cette commune attenant à l'église insultant journellement le prêtre dans ses fonctions du sacerdoce et en faisant chanter par ses ouvriers les chansons les plus obscènes [...] ${ }^{17}$. Bref, les partisans de Saint-Just sont plus nombreux à la mairie qu'à l'église, d'autant que ses connaissances juridiques lui permettent de proposer gracieusement ses services à ses compatriotes dans l'affaire des communaux.

L'affaire des communaux

19 Elle a été le catalyseur essentiel du mécontentement. Elle exaspère en effet de nombreux habitants, d'autant qu'elle s'accompagne de l'interdiction d'emprunter des chemins fréquentés jusque-là librement depuis des temps immémoriaux. Le 17 octobre 1790, Saint-Just est mandaté par quarante-huit de ses compatriotes pour conférer de la question avec le seigneur Grenet ${ }^{18}$. Mais celui-ci, en réponse du 16 novembre ${ }^{19}$ et visiblement dans l'intention de gagner du temps, d'entretenir la confusion, propose d'aboutir à une transaction amiable négociée avec une délégation communale composée de douze personnes, dont plusieurs gros laboureurs eux-mêmes spoliateurs... On ne s'étonnera pas du silence qui s'en suit. Il faut attendre le 7 août 1791 pour que Saint-Just reçoive mission de porter l'affaire au bureau de conciliation de Coucy-leChâteau et le 7 novembre suivant pour que le district autorise la commune à poursuivre Grenet en justice, ce qui, à vrai dire, voue la cause à l'échec, car une procédure orthodoxe impliquerait que les requérants eussent fait procéder à un arpentage coûteux et eussent été en mesure de rembourser les investissements engagés par les spoliateurs. Or, de tels frais étaient totalement hors de portée d'une commune aussi impécunieuse que celle de Blérancourt. Le contentieux ne s'évanouira que dans le temps long. Ni la Révolution violente, ni l'Empire n'y mettront un terme, puisque Grenet réclame encore ses bois par exploit d'huissier le 28 décembre 1814 et, après sa mort, ses héritiers suivront la même voie ${ }^{20}$. Saint-Just n'y perd néanmoins pas la face, car la courbe ascendante de sa carrière politique et le tour que prend la Révolution 
laissent à ses amis l'impression d'un succès. La tentative de fédération communale est moins réussie.

Fédération de communes

20 La victoire à la mairie de Blérancourt avait été, on l'a vu, le résultat d'une audace légalement douteuse, mais contre laquelle l'administration n'a pas réagi, probablement exaspérée par l'activisme de Gellé qui inonde les autorités de protestations, jusqu'à l'Assemblée nationale à Paris. Grâce à Victor Thuillier, secrétaire-greffier et Louis Honnoré, maire jusqu'à la fin de l'année 1791, Saint-Just y exerce une influence prépondérante. Et c'est sans doute pour élargir son audience dans le même registre qu'il essaye d'exporter ses méthodes afin de placer à la tête des communes voisines des conseils généraux favorables aux idées nouvelles, voire de constituer une fédération.

21 Son dévolu se porte sur Manicamp, gros village surpeuplé sis au confluent de l'Oise et de l'Ailette, à une lieue et demie de Blérancourt et dirigé comme presque partout par une municipalité occupée par les plus gros propriétaires. Une première démarche, consignée au registre ${ }^{21}$, est celle de cinquante-quatre habitants de Manicamp qui se rendent à Blérancourt et demandent aux officiers municipaux leur médiation et leur présence lors de la formation d'une nouvelle municipalité, vu que la leur n'est, selon eux, pas représentative. Après en avoir délibéré, le conseil municipal sollicité se transporte, le 27 juin 1790, à l'église de Manicamp où l'attendent cent-vingt-cinq citoyens qui procèdent aussitôt à l'élection d'un maire, d'un procureur, d'officiers municipaux et de douze notables. Le procès verbal se termine par une cascade de griefs à l'encontre de la représentation officielle qui se serait autoproclamée quelques mois plus tôt et agit depuis sans légitimité. Le nouveau conseil est validé par cent vingtquatre citoyens : soixante-dix ont apposé des signatures pour la plupart grossières, à peine lisibles, et cinquante-quatre, dûment nommés, ont déclaré ne savoir signer. Presque tous sont qualifiés de tisserands ou nourtiers. Il s'agit incontestablement des plus pauvres habitants du village.

Cette municipalité de va-nu-pieds ne siégera du reste jamais, car les autorités n'hésiteront pas cette fois à casser ces élections improvisées. Malgré les menaces, il n'y aura pas de suites judiciaires. Ce n'en est pas moins un grave revers pour Saint-Just qui avait probablement rêvé de fédération pour élargir sa représentativité. Du moins lui reste-t-il les ressources de la garde nationale pour accéder aux mêmes objectifs.

La garde nationale

23 Une tradition tenace veut que Saint-Just ait commandé la garde nationale du village avec le grade de lieutenant-colonel et qu'il en ait fait son instrument. La réalité est beaucoup plus nuancée. Le nom de Saint-Just n'apparaît ni dans les cadres nommés, ni dans les signataires de l'acte constitutionnel de la garde, le 7 février $1790{ }^{22}$. On ne le voit pas non plus, sinon comme témoin extérieur, prendre part aux turbulences picaresques qui agitent la petite société des citoyens-soldats de Blérancourt pendant tout le printemps ${ }^{23}$, même si ses interventions occultes sont évidentes. Le registre n'associe son nom à l'activité de cette troupe villageoise que le 6 juin 1790, à l'occasion d'une réception de trois citoyens dans la garde ${ }^{24}$ : Warnier comme lieutenant, Carbonnier fils comme chirurgien major et Saint-Just comme lieutenant-colonel. Mais alors que le texte a été respecté dans la relation de réception des deux premiers, il est totalement caviardé et rendu illisible quand il s'agit de Saint-Just, sans qu'on puisse savoir si cette intervention est contemporaine, au motif qu'il n'a pas l'âge requis pour être citoyen actif, ou plus tardive. On serait tenté de penser qu'elle est tardive, car le 2 
juillet 1790, lors de la nomination d'une députation de la garde à Chauny et, un an plus tard le 24 juin 1791, lors de l'envoi d'un détachement chargé d'aller à la rencontre du roi au retour de sa fuite, Saint-Just est désigné sous ce grade. Cela ne peut néanmoins être considéré comme une preuve irréfutable dans la mesure où c'est Thuillier qui tient la plume du greffe... Mais il est incontestable que, pour des raisons évidentes de stratégie, Saint-Just a cherché à être reconnu comme lieutenant-colonel, car ce grade l'eût situé au-dessus de tous les commandants de bataillon, c'est-à- dire en tenant compte du peu d'assiduité du colonel Decaisne, son beau-frère, au sommet de la hiérarchie de la garde. C'est ainsi que le 4 mars 1792, il fait encore suivre sa signature au procès-verbal des deux lettres L.C. ${ }^{25}$, en toute illégitimité comme en témoigne le renouvellement électoral intervenu le mois précédent. Le 5 février 1792, en effet, la garde nationale est répartie en trois divisions de quatre-vingts à quatre-vingt-cinq hommes et Saint-Just est élu capitaine de la troisième. Une semaine plus tard, le 12, nouveau scrutin pour former «l'état-major des bataillons » au cours duquel il est élu " commandant en second du bataillon de Blérancourt », sous l'autorité de Le Vasseur, propriétaire au village voisin de Saint-Aubin, élu "commandant en chef». Et c'est encore comme "commandant en second " qu'il représente la garde à la fête de la fédération du 14 juillet 1792 à Chauny ${ }^{26}$.

Par ailleurs, et comme le clan Gellé l'avait fait avant lui, ${ }^{27}$ Saint-Just a tenté d'épurer la garde nationale en écartant les éléments hostiles à la Révolution. On croirait l'entendre lorsque, le 15 juin $1790{ }^{28}$, sont définies les conditions d'admission: les candidats devront se présenter devant le conseil général de la commune qui examinera «si leur zèle n'est point vanité, si sous les détours de l'humilité ils ne cachent point l'intrigue et la duplicité et si leur retour n'est pas un nouveau moyen de tromper plus adroitement le bien public », et c'est le certificat du conseil " qui leur servira de titre d'admission » 29. Afin de ne pas entraver le volontariat de ceux qui, comme Saint-Just ou beaucoup de ses amis, n'ont pas l'âge légal requis de 25 ans ou ne remplissent pas les conditions de cens (contribution de trois journées de travail) pour être citoyen actif, le conseil général en donne son interprétation: «[...] pour être citoyen actif, l'assemblée nationale entend surtout un homme revêtu de la sanction de tous ses concitoyens et ne leur défend pas d'exclure, par une sage politique, ceux qui portent avec eux le poison de l'aristocratie ; voilà l'esprit de ses écrits et nous avons juré mille fois de leur être fidèles ». Un esprit, il faut en convenir, assez loin de la lettre !

En fait, Saint-Just ne parviendra pas à imposer durablement son autorité sur la garde qui est loin d'être un instrument entre ses mains - surtout dans le cadre du canton où il ne dispose pas d'autant de fidélités qu'au village - et le commandement lui en est contesté jusqu'au bout. Paradoxalement, le bilan paraît peu positif au plan local où les vaines poursuites contre Gellé ne se soldent que par de coûteuses démarches aux frais de la commune et où les prometteuses tentatives de fédérations ${ }^{30}$ restent finalement sans lendemain. Cet activisme un peu brouillon permet néanmoins au futur Conventionnel de maintenir son camp en état de mobilisation et de faire connaitre son ardente foi révolutionnaire jusqu'à l'Assemblée constituante où la France partagea un instant l'émotion de ces paysans de Blérancourt détruisant par le feu un "infâme " libelle contre-révolutionnaire ${ }^{31}$. Nul doute pourtant que le soldat-citoyen Saint-Just n'ait trouvé dans les difficultés à s'imposer matière à réflexion sur l'autorité et que le défenseur officieux des intérêts du village n'ait profité de l'expérience acquise dans l'affaire des communaux. 
Sans bouleverser les conclusions de deux siècles d'historiographie, ce document apporte d'intéressantes précisions. L'observation minutieuse des écritures, ratures, surcharges et corrections de la main de Saint-Just confirme son rôle capital dans l'animation politique de Blérancourt et éclaire les principaux traits de son caractère : sens de la communication, de l'imagination, de la dissimulation (minorité oblige!), disponibilité, extraordinaire énergie, opiniâtreté, la main sans cesse sur le pommeau de l'épée et toujours en avance d'une guerre, il apparaît en 1792 comme un battant, un anti-fataliste, le contraire en somme du Saint-Just de Thermidor.

Ce document conforte aussi la nature formatrice du milieu. Les décrets et arrêtés de l'Assemblée nationale sont recopiés sur les registres par pages entières. La rhétorique des décisions communales montre que le jeune homme les a lus, étudiés, disséqués, assimilés pour les appliquer. Il y a appris la fidélité et la trahison, la duplicité, les joies des petites victoires et les déconvenues du lendemain, les mystères de l'autorité. Paraphrasant un mot célèbre, on pourrait dire qu'il a fait son apprentissage à l'université de Blérancourt.

Enfin, le document éclaire la nature de la pauvreté au service de laquelle il se place. Ses pauvres sont moins les sans-culottes parisiens que ceux au milieu desquels il a vécu. Ceux-ci appartiennent à une sorte de quart état constitué de jeunes, de démunis, d'illettrés, d'exclus, d'hommes et de femmes sans ressources et sans avenir. Ses pauvres sont les manouvriers de Blérancourt, les tisserands et les nourtiers de Manicamp dont il aurait voulu faire des citoyens en leur donnant un champ, alors gage de survie dans la dignité. Ce sont eux, ces "puissances de la terre", qui ont inspiré les décrets de ventôse et ce projet n'est pas, du moins pour Saint-Just, une manœuvre de manipulation à l'usage de la rue, mais une mesure propre à contribuer au soulagement de la misère et à appliquer d'urgence, comme en témoignent les corrections qu'il porte de sa main sur la minute du texte et les efforts qu'il fait jusqu'à son dernier jour pour tenter de les appliquer ${ }^{32}$.

\section{NOTES}

1.Charles DESSIN, Le bourg de Blérancourt, Saint-Quentin, 1926.

2.Sous la cote : A.C. Blérancourt, 1 D 1.

3.R.D.M.B. (registre des délibérations municipales de Blérancourt), 2e registre, 33e et $34 \mathrm{e}$ feuillets. L'arpent local équivaut à environ 41 ares.

4.Ibid., 1er registre, 11e feuillet.

5.A. D. Aisne, C 1022 au 14 mai 1788 et C 910 au 22 mai 1788.

6.L'essain de Soissons équivaut à 51,24 litres.

7.B. VINOT, Saint-Just, Paris, Fayard, 1985, p.85.

8.On sait que cette crainte motive la première lettre de Saint-Just à Robespierre le 19 août 1790.

9.R.D.M.B. 1er registre, $18 \mathrm{e}$ feuillet.

10.Ibid., 24e feuillet recto. 
11.Ibid., 24e feuillet verso : « [...] les dits syndics et officiers municipaux soussignés n'ont pas cru devoir de considération à l'arrêté du jour d'hier qui prolonge sans raison le terme de la convocation, déclarent le dit arrêté nul et de nulle valeur attendu qu'il altère les dispositions du règlement qui fixe l'assemblée de la commune à huit jours de la date de la publication ».

12.Ibid., 28e feuillet verso.

13.Ibid., Cette feuille est conservée entre le 2e et le $3 e$ registre et recopiée sur le $2 \mathrm{e}$ registre, $38 \mathrm{e}$ feuillet recto.

14.Ibid., 1er registre, $28 \mathrm{e}$ feuillet, recto.

15.Ibid., 2e registre, $20 \mathrm{e}$ feuillet recto et verso.

16.Ibid., 66e feuillet verso.

17.A.N., F7 67881 à 60. Je remercie vivement Laurent Brassard qui m'a communiqué ce document.

18.R.D.C.B., 2e registre, $37 \mathrm{e}$ feuillet recto.

19.Ibid., 42e feuillet recto.

20.A.D. Aisne ; A. C. Blérancourt, 1 D 2.

21.Sur cette affaire, voir R.D.C.B., 2e registre, 16, 17, 18e feuillets.

22.R.D.M.B., 1er registre, 25e feuillet verso.

23.B. VINOT, op. cit., pp.89-91.

24.R.D.M.B., 2e registre, $11 \mathrm{e}$ et $12 \mathrm{e}$ feuillet.

25.Ibid., 3e registre, 13e feuillet, recto.

26.Ibid., 20e feuillet recto.

27.Voir supra note 9.

28.R.D.C.B., 2e registre, 13e feuillet recto et verso.

29.Ibid. Un autre arrêté à la date du 20 juin va dans le même sens : « ...toute incorporation sera nulle si le sujet n'y est présenté par le procureur de la commune et s'il n'est point admis par le suffrage de tous les officiers attendu que nul n'est maître absolu dans sa compagnie et que le corps tout entier doit être consulté ».

30.B. vinot, op. cit., pp.89-94.

31.Id., p.86. SAINT-JUST, Euvres complètes, éd. établie et présentée par A. KUPIEC et M. ABENSOUR, Paris, Gallimard folio histoire, 2004.

32.Id., pp. 284-285.

\section{RÉSUMÉS}

La redécouverte des registres de délibérations communales de Blérancourt (Aisne) portant sur la période 1788-1793 permet de suivre la pré-Révolution et les quatre premières années de la Révolution dans ce bourg de mille habitants comprenant une seigneurie, un monastère de Feuillants et une influente paroisse. Dès le début de l'année 1790, la vie politique y est animée par Saint-Just. Celui-ci parvient à entraîner une petite moitié des habitants (dont 15\% de femmes) dans la lutte contre la seigneurie et l'Église en s'appuyant dans les mairies et la garde nationale sur quelques sans-culottes et sur la partie la plus indigente de la population. Les résultats sont 
mitigés au plan pratique, mais de première importance pour la formation et l'engagement politique du futur Conventionnel.

Revolution in the Village, with Saint-Just, based on the Municipal Records of Blérancourt. The rediscovery of the minutes of the municipal deliberations of Blérancourt (Aisne) for the period 1788-1793 sheds light on the pre-revolutionary period and the first four years of the Revolution in this small town of one thousand inhabitants, comprising a manor, a Feuillant monastery and an influential parish. From early 1790, political life found a leader in Saint-Just. He succeeded in motivating about half of the locals (including $15 \%$ of women) in the struggle against the manor and the church, relying on a handful of sans-culottes in the town-hall and the national guard, and on the poorest portion of the population. The results were scant from a practical viewpoint, but of great importance in terms of the political development and commitment of the future member of the Convention.

INDEX

Mots-clés : Révolution montagnarde, Saint-Just, Blérancourt, Révolution et féminisme, Révolution et Église, biens communaux 